\title{
ASSOCIATION OF LIPID PROFILES WITH 10-YEAR ATHEROSCLEROTIC CARDIOVASCULAR DISEASE RISK: STUDY AMONG SUBJECTS IN SLEMAN DISTRICT OF YOGYAKARTA INDONESIA
}

\author{
RITA SUHADI*, DITA MARIA VIRGINIA, CHRISTIANUS HERU SETIAWAN
}

Department of Pharmacology and Clinical Pharmacy, Faculty of Pharmacy, Sanata Dharma University, Jl. Affandi Yogyakarta 55002, Indonesia. Email: ritasuhadi@usd.ac.id

Received: 19 June 2017, Revised and Accepted: 24 August 2017

\section{ABSTRACT}

Objectives: The subjects in the Sleman District of Yogyakarta had medium Framingham risk score (FRS) in the preceding year study. The 10-year atherosclerotic cardiovascular disease (ASCVD) risk was a newer risk estimator than FRS. This study aimed to associate the lipid profiles with the ASCVD risk

Methods: The study was conducted with a cross-sectional design and the subjects were selected with cluster random sampling. The association of lipid profiles and ASCVD risk was analyzed with Mann-Whitney/Kruskal-Wallis tests and Spearman's rho correlation, whereas the categorical scores within sub-groups were analyzed Chi-square statistics, respectively.

Results: The eligible subjects $(\mathrm{n}=221)$ had the age at $51.7 \pm 8.1$ years, systolic/diastolic blood pressure $136.8 \pm 22.4 / 85.0 \pm 12.4 \mathrm{mmHg}$, total-cholesterol (total-C), high-density lipoprotein-cholesterol (HDL-C), low-density lipoprotein-cholesterol (LDL-C), and triglyceride at $202.0 \pm 34.9$ mg/dl, $52.6 \pm 12.5 \mathrm{mg} / \mathrm{dl}, 126.3 \pm 30.0 \mathrm{mg} / \mathrm{dl}$, and $135.9 \pm 95.4 \mathrm{mg} / \mathrm{dl}$, respectively; with hypertension treatment $16.7 \%$, smoking $52.9 \%$, diabetes $10.4 \%$, and the median ASCVD risk at 4.4 (0.2-41.4). The ASCVD risk has significant association with non-lipid profiles, total-C, lipid ratio of triglyceride/HDL-C, total-C/HDL-C, and LDL-C/HDL-C, to a lesser extent, HDL-C, LDL-C, and triglyceride.

Conclusion: The 10-year ASCVD risk of the subjects was categorized as low and had a significant association with total-C and lipid ratio of triglyceride/ HDL-C, total-C/HDL-C, and LDL-C/HDL-C

Keywords: Atherosclerotic cardiovascular disease, 10-year atherosclerotic cardiovascular disease risk, lipid profiles, cholesterol, ratio totalcholesterol/high-density lipoprotein-cholesterol-C, ratio low-density lipoprotein-cholesterol/high-density lipoprotein-cholesterol.

(C) 2017 The Authors. Published by Innovare Academic Sciences Pvt Ltd. This is an open access article under the CC BY license (http://creativecommons. org/licenses/by/4. 0/) DOI: http://dx.doi.org/10.22159/ajpcr.2017.v10i12.20675

\section{INTRODUCTION}

Cardiovascular diseases (CVDs) cause the global highest mortality rate [1]. The CVDs are caused by the cumulative risk factors including obesity, hypertension, hypercholesterolemia, diabetes mellitus (DM), smoking, unhealthy lifestyle, race, and education [2,3]. A newer guideline on the hypercholesterolemia treatment to reduce "atherosclerosis cardiovascular risk in adults (atherosclerotic cardiovascular disease [ASCVD]]" was introduced in the year 2013 by American College of Cardiology/American Heart Association (ACC/AHA). In the conventional guideline, the total-cholesterol (total-C) levels at $<200 \mathrm{mg} / \mathrm{dl}$ and $\geq 240 \mathrm{mg} / \mathrm{dl}$ were categorized as desirable and high, respectively; the high-density lipoprotein-cholesterol (HDL-C) levels at $<40 \mathrm{mg} / \mathrm{dl}$ and $>60 \mathrm{mg} / \mathrm{dl}$ were categorized as low and high; and the low-density lipoprotein-cholesterol (LDL-C) levels at $<100 \mathrm{mg} / \mathrm{dl}$ and $>160 \mathrm{mg} / \mathrm{dl}$ as were as ideal high [4]. The newer guideline is substantially different from the conventional one because the guideline did not provide the exact cholesterol cut point for CVDs [5].

A terminology of ASCVD replaced the established term of CVD [5]. The 10-year ASCVD risk for the first cardiovascular event in percentage could be predicted with several methods, e.g., systemic coronary risk estimation (SCORE) used among European countries, Framingham risk score (FRS), pooled cohort equation (PCE) with the 10-year ASCVD risk, and some other methods [6,7]. The ASCVD risk showed moderate discrimination and good calibration in an Asian population. The moderate discrimination due to the high prevalence of ASCVD risk factors was improved with clustering of higher risk patients, whereas fewer patients were needed among the patients with lower risk [8]. Therefore in a general population of subjects with a wider range of
ASCVD risk, the risk prediction using PCE method may give a better discrimination $[8,9]$

The 10-year ASCVD risk of PCE was obtained from four pooled cohort prospective studies including Framingham studies both original and derivative studies with $>20,000$ subjects aged at 20-79 years [2]. The PCE estimates the hard risk of ASCVD including myocardial infarction, coronary hard disease death, nonfatal, and fatal stroke $[6,10]$.

In the 2013 ACC/AHA Guideline, the initiation and continuing treatment for hypercholesterolemia was determined by the 10-year ASCVD risk score, the existence of DM, and LDL-C level. Furthermore, a statin was the only beneficial medication for the hard ASCVD reduction in hypercholesterolemia in the guideline [5,6]. The conventional FRS method has the cardiovascular risk classification of $>20$ (high), 10-20 (moderate), and $<10$ (low) of 10-year risk [4], while the 10-year ASCVD risk has the classification as follow: $<5,5-<7.5, \geq 7.5-<10$, and $\geq 10$ as low, moderate, high, and very high risk, respectively [11]. While the 10-year ASCVD risk was implemented in the screening of the four statin-favored subjects, the risk was only classified into three categories, i.e., $<5,5-<7.5, \geq 7.5$ as low, moderate, and high risk. The four statin-favored subjects consist of (1) clinical atherosclerosis, (2) DM, (3) LDL-C higher than $190 \mathrm{mg} / \mathrm{dl}$, and (4) subjects without DM or ASCVD but with $\geq 7.5$ of 10-year ASCVD risk [5,9].

The subjects in the Sleman District ( $\mathrm{n}=926)$ in the year 2015 were reported to be high prevalence of hypertension but with low awareness and therapy of the disease [12], meanwhile from the study conducted in the district in the year 2016, the subjects $(n=222)$ had the FRS at $11.4 \pm 8.9$, and classified as medium risk [13]. There was no information 
regarding the 10-year ASCVD risk using PCE method found among the population in Sleman District. This study aimed to investigate the 10-year ASCVD risk using the PCE method and to associate the ASCVD risk score with the lipid profile of the subjects.

\section{METHODS}

This study was the year two survey with the focus on the therapeutic lifestyle changes among the subjects in Sleman District. The study was an observational research conducted with analytical cross-sectional design. The sites of study were selected with stratified random sampling. The protocol has been approved with the ethical clearance Ref No: KE/FK/043/EC/2016 by Ethics Committee of Gadjah Mada University, Yogyakarta. The preceding year study has been published as: "The Metabolic Disorder and Cardiovascular Risk among Lower Socioeconomic Subjects" [13]. The FRS risk score from the preceding year study was not compared with the ASCVD risk from this study. The subjects between studies were different because the excluded subjects from year one due to the failure of meal fasting were invited as the subjects of the year two study. Moreover, the FRS method includes a wider range of age and total-C than the ASCVD risk and covers more subjects accordingly. Data in year two were added with LDL-cholesterol and triglyceride measurement.

The subjects were selected with cluster random sampling from the study sites. All subjects met the criteria of 10-year ASCVD risk were included. The criteria of PCE method included gender (male and female), age within 40-79 years old, HDL-C within 20-100 mg/dl, total-C at $130-320 \mathrm{mg} / \mathrm{dl}$, systolic blood pressure (SBP) within $90-200 \mathrm{mmHg}$, race (white, African-American, or others), diabetes (yes, no), treatment for hypertension (yes, no), and smoker (yes, no). Exclusion criteria in year one study covered the subjects with hard ASCVD and/or failed to proceed the 8-10 hrs meal fasting.

\section{Analysis}

The ASCVD risk calculator from PCE method is available online for two types of ASCVD risks, i.e.: 10-year risk for subjects at the age 40-79 years and lifetime ASCVD risk for the age at 30-60 years [9]. The study focused more on 10-year ASCVD risk of the 236 eligible participants for PCE at the age of 30-75 years; there were 15 subjects could not be calculated due to the age $<40$ years old, but they were included in the lifetime ASCVD risk score calculation. The cut point of the lipid profile used the standard of the National Cholesterol Education Program Expert Panel on Detection, Evaluation, and Treatment of High Blood Cholesterol in Adults (Adult Treatment Panel III) guideline [4].

Before the evaluation of the variable association, the data were analyzed for the normality of data distribution and the body mass index (BMI) was the only normally distributed variable. The subgroup difference of the 10-year ASCVD risk within a variable was analyzed with Mann-Whitney U-test or with Kruskal-Wallis test due to the data distribution. The association of 10-year ASCVD risk in categorical risk with the lipid profile in categorical risk was analyzed with $2 \times 2$ Chi-square test. The lowest cut point sub-group in each variable was determined as a control group. In the Chi-square analysis, the 10-year ASCVD risk was grouped into two classifications, i.e., lower risk at $<7.5$ and higher risk at $\geq 7.5$ ASCVD risk. The variables of PCE method were correlated with the ASCVD risk score using the Spearman's Correlation analysis, and the coefficients (rho or r) at $<0.3, \geq 0.3-<0.7$, and $\geq 0.7$ were categorized as weak, moderate, and strong correlation [14].

\section{RESULTS}

Of the total subjects $(n=236)$, the eligible subjects for 10-year ASCVD risk were 221 respondents, whereas the eligible subjects for lifetime risk method were 182 respondents. The study focused only the 10 -year ASCVD risk in the further discussion. The median 10-year ASCVD risk score was at $4.4 \%$ and classified as low-risk ( $<5$ score), and the risk classified the subjects into four levels of risk, i.e., low, moderate, and high-risk subjects [11], and most subjects were in the low-risk. Although most of the subjects had low ASCVD risk, overall there 81 subjects (36.7\%) needed statin according to guideline. The statin was needed for the DM subjects $(n=23)$ distributed into low-risk $(n=9)$, moderate risk $(n=4)$, and high-risk $(n=14)$, and among those without DM ( $n=58)$ but with $\geq 7.5$ ASCVD risk (Fig. 1) [5].

The subject characteristics were not normally distributed except for BMI, and the data were presented as median value. The subjects had relatively young age at median 51 years. The subject characteristics including SBP/diastolic blood pressure (DBP), pulse, blood glucose, and lipid profiles showed the near ideal to borderline median values (Table 1).

The parameter of ASCVD variables, i.e., gender, treatment for hypertension, DM, smoking, age, systolic blood pressure (SBP), and BMI was classified into the subgroups based on the categorical risk. The lowest cut point subgroup in each variable acted as the control group. The result showed the association all estimators with the 10-year ASCVD risk significantly $(\mathrm{p}<0.05)$ with Mann-Whitney/Kruskal-Wallis test. The ASCVD risk was not associated with BMI $(\mathrm{p}=0.60)$. The increased risk in each variable showed the increased odds ratio related to ASCVD risk, i.e., in male, older age, higher SBP, the existence of DM, and hypertension treatment, but excluding the smoking and BMI variables.

The correlation between the ASCVD and the ASCVD variables plus BMI was done using the Spearman's test. The BMI was not belonged to the ASCVD variables, but it was included in the analysis because BMI is an ASCVD risk in general population. The age variable had the strongest correlation with the ASCVD risk followed by the SBP with moderate correlation. The variable of age had the strongest correlation with ASCVD at moderate intensity $(r=0.67)$ with the ASCVD risk among all

Table 1: Characteristics of cardiovascular risk and lipid profiles

\begin{tabular}{ll}
\hline $\begin{array}{l}\text { Characteristics of cardiovascular } \\
\text { risk estimators and 10-year risk } \\
\text { score (n=221) }\end{array}$ & $\begin{array}{l}\text { Mean } \pm \text { SD } \\
\text { median } \\
\text { (minimum-maximum) }\end{array}$ \\
\hline Age (years old) & $51.7 \pm 8.1$ \\
& $51(40-73)$ \\
Systolic blood pressure or SBP (mmHg) & $136.8 \pm 22.4$ \\
& $133(101-200)$ \\
Diastolic blood pressure or & $85.0 \pm 12.4$ \\
DBP (mmHg) & $84(54-126)$ \\
Pulse (x/minute) & $80.9 \pm 13.9$ \\
Body mass index or BMI kg/m² & $79(46-129)$ \\
& $24.2 \pm 4.5^{*}$ \\
Fasting blood glucose or FBG (mg/dl) & $24.1(14.2-41.4)$ \\
& $98.1 \pm 50.0$ \\
10-year ASCVD risk (\%) & $85(59-565)$ \\
& $7.3 \pm 7.4$ \\
Total-cholesterol (mg/dl) & $4.4(0.2-41.4)$ \\
HDL-cholesterol (mg/dl) & $202.0 \pm 34.9$ \\
& $197.0(127-320)$ \\
LDL-cholesterol (mg/dl) & $52.6 \pm 12.5$ \\
& $51(26-96)$ \\
Triglyceride or TG (mg/dl) & $126.3 \pm 30.0$ \\
Ratio TG/HDL (mg/dl) & $124(45-225)$ \\
& $135.9 \pm 95.4$ \\
Ratio LDL/HDL (mg/dl) & $109(43-1018)$ \\
Ratio total-cholesterol/HDL (mg/dl) & $3.0 \pm 2.9$ \\
& $2.2(0.5-29.1)$ \\
& $2.5 \pm 0.9$ \\
& $2.6(1.0-7.1)$ \\
& $3.9(2.0-10.3)$ \\
\hline
\end{tabular}

*Data: Normally distributed. ASCVD: Atherosclerotic cardiovascular disease, HDL: High-density lipoprotein, LDL: Low-density lipoprotein, SD: Standard deviation 
variables of lipid or non-lipid profiles, and the correlation with SBP was at the second highest, the correlation with smoking, and gender variables; out of these four variables the other variables had the weak correlation (Table 2)

Similar to non-lipid profiles, the categorical lipid profiles were significantly different using the Kruskal-Wallis test $(p<0.01)$. The higher

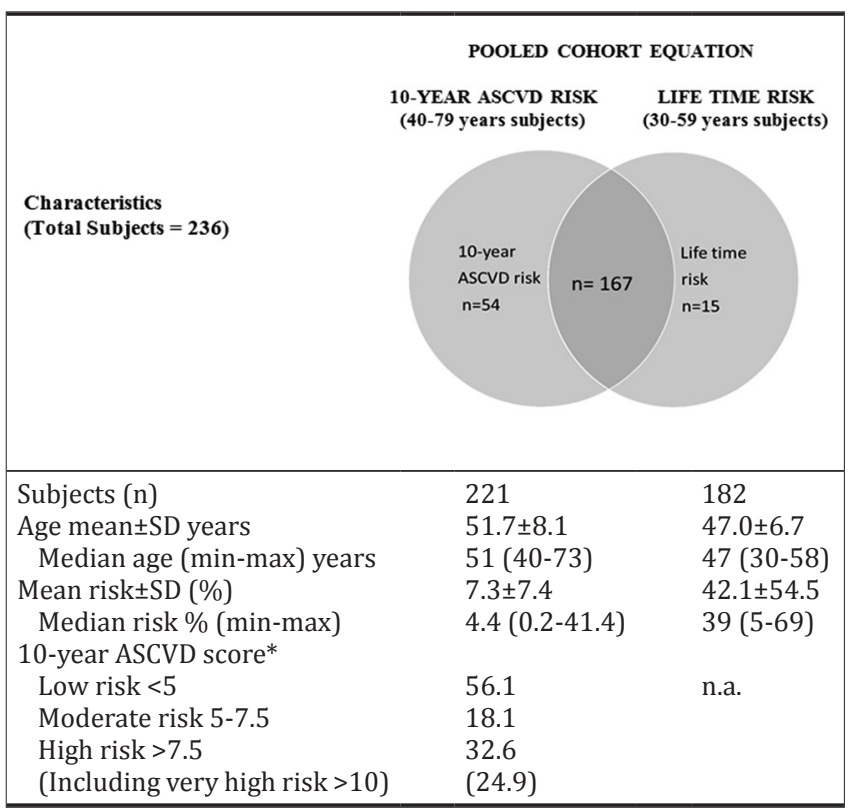

Fig. 1: The subjects distribution with 10-year risk score and lifetime risk score using pooled cohort equation method. *Diabetes mellitus (DM) comorbid subjects $(n=27)$ : Atherosclerotic cardiovascular disease (ASCVD) risk $\geq 7.5$ $(n=14) ;>5-<7.5(n=4) ;<5(n=9)$; without DM $(n=58)$ but with $\geq 7.5$ ASCVD risk
total-C in categorical classification had the higher ASCVD risk, whereas the lowest HDL-C sub-group has the highest ASCVD risk $(\mathrm{p}<0.05)$. The total-C, ratio of triglyceride/HDL-C, total-C/HDL-C, and LDL-C/HDL-C had significantly different of 10-year ASCVD risk in Mann-Whitney test in all sub-groups, whereas the HDL-C, LDL-C, and triglyceride had significantly different of risk in some sub-groups. The two lowest subgroups of LDL-C; HDL-C; and total-C had not-significantly different 10-year ASCVD risk within the variables. All lipid profile had a weak correlation with 10-year ASCVD risk (Table 3).

\section{DISCUSSION}

The finding of the low 10-year ASCVD risk this study was different from the previous year result using the FRS method in the moderate risk [13]. The difference was likely caused by the inclusion of subjects. The ASCVD risk of the PCE could not comprehensively include all subjects from FRS method. The ASCVD risk method was limited for the subjects with 40-79 years, total-C of 130-320 mg/dl, and HDL-C of 20-100 mg/dl [3,9].

In this study, all subjects in the cluster were invited, and the subjects in the subgroups could not be matched for all of the nine variables in 10-year ASCVD risk, e.g., fewer male, DM, and with therapy subjects. In general population, the ASCVD risk is higher in male than female subjects, though the gender risk difference diminished in the elderly subjects [15], the ASCVD risk is also higher among DM and/or with hypertension therapy [9]. The similar findings from this study showed that the male, DM, and/or with hypertension therapy subjects had a significantly higher risk.

The low ASCVD risk was likely related to the subjects' age because the age had the strongest correlation to the ASCVD risk among other variables. The ASCVD risk in this study had much higher correlation than the FRS and age correlation found in a study done among the outpatients found in Baghdad with $\mathrm{r}=0.296$ [16].

The median age of the subjects at 51 years was younger than the age with ASCVD risk at 55 years. The ASCVD history occurred at $<55$ and $<65$ years for male and female, respectively, was defined as

Table 2: Distribution of the 10-year ASCVD using risk pooled cohort equation risk estimators for the cardiovascular disease-related variables

\begin{tabular}{|c|c|c|c|c|c|}
\hline Characteristics & $\begin{array}{l}\text { 10-year CVD risk } \\
\text { Mean } \pm \text { SD }\end{array}$ & $p$ value $e^{* 1}$ & $\begin{array}{l}\text { Spearman's rho }(\mathrm{r}) \\
\text { correlation*2 }\end{array}$ & Proportion n (\%) & OR (CI 95\%)*3 \\
\hline \multicolumn{6}{|l|}{ Gender } \\
\hline Male & $12.8 \pm 9.3$ & - & \multirow[t]{2}{*}{-0.34} & $52(23.5)$ & 1.0 \\
\hline Female & $5.5 \pm 5.7$ & $\mathrm{p}<0.01$ & & $169(76.50$ & $0.18(0.10-0.35)$ \\
\hline \multicolumn{6}{|c|}{ Hypertension treatment } \\
\hline Yes & $12.5 \pm 9.1$ & - & \multirow[t]{2}{*}{-0.27} & 37 (16.7) & 1.0 \\
\hline No & $6.2 \pm 6.5$ & $\mathrm{p}<0.01$ & & $184(83.3)$ & $0.2(0.1-0.4)$ \\
\hline \multicolumn{6}{|l|}{ Diabetes mellitus } \\
\hline Yes & $14.9 \pm 11.7$ & - & \multirow[t]{2}{*}{-0.20} & $23(10.4)$ & 1.0 \\
\hline No & $6.4 \pm 6.1$ & $\mathrm{p}<0.01$ & & $198(83.3)$ & $0.3(0.1-0.6)$ \\
\hline \multicolumn{6}{|l|}{ Smoking } \\
\hline Yes & $9.5 \pm 8.2$ & - & \multirow[t]{2}{*}{-0.21} & $117(52.9)$ & 1.0 \\
\hline No & $4.8 \pm 5.2$ & $\mathrm{p}<0.01$ & & $104(47.1)$ & $0.57(0.32-1.00)$ \\
\hline \multicolumn{6}{|l|}{$\operatorname{Age}^{* 4}$} \\
\hline$\leq 50$ years & $3.5 \pm 4.2$ & - & \multirow[t]{2}{*}{0.67} & $117(52.9)$ & 1.0 \\
\hline$>50$ years & $10.7 \pm 7.9$ & $\mathrm{p}<0.01$ & & $104(47.1)$ & $15.1(7.0-32.6)$ \\
\hline$<140 \mathrm{mmHg}$ & $4.2 \pm 4.1$ & - & \multirow[t]{4}{*}{0.53} & $133(60.2)$ & 1.0 \\
\hline $140-<160 \mathrm{mmHg}$ & $10.0 \pm 6.3$ & $\mathrm{p}<0.01$ & & $52(23.5)$ & $6.5(3.2-13.4)$ \\
\hline $160-<180 \mathrm{mmHg}$ & $13.8 \pm 8.5$ & $\mathrm{p}<0.01$ & & $26(11.8)$ & $22.5(8.0-63.0)$ \\
\hline$\geq 180 \mathrm{mmHg}$ & $16.4 \pm 11.1$ & $\mathrm{p}<0.01$ & & $10(4.5)$ & $15.7(3.7-66.1)$ \\
\hline \multicolumn{6}{|l|}{$\mathrm{BMI}^{* 4}$} \\
\hline$<24.3 \mathrm{~kg} / \mathrm{m}^{2}$ & $6.7 \pm 6.2$ & - & \multirow[t]{2}{*}{-0.03} & 110 (49.8) & 1.0 \\
\hline$\geq 24.3 \mathrm{~kg} / \mathrm{m}^{2}$ & $7.9 \pm 8.3$ & $\mathrm{p}=0.60$ & & $111(50.2)$ & $1.06(0.61-1.84)$ \\
\hline
\end{tabular}

CVD: Cardiovascular disease; 10 -year risk in percentage (\%). ${ }^{* 1} \mathrm{p}$ value: Mann-Whitney/Kruskal-Wallis for 2 or 3 sub-group variables. $\mathrm{p}$ value compared with the lowest cut point sub-group. ${ }^{* 2}$ Correlation of the variables with 10 -year ASCVD risk. ${ }^{* 3}$ OR (95\% CI): Odds ratio ( $95 \%$ confidence interval); OR compared with the lowest cut point sub-group. ${ }^{* 4}$ Age and BMI sub-group cut point based on median value. BMI: Body mass index, ASCVD: Atherosclerotic cardiovascular disease, SD: Standard deviation 
Table 3: Distribution of the 10-year ASCVD using risk pooled cohort equation risk estimators for lipid profiles

\begin{tabular}{|c|c|c|c|c|c|}
\hline Characteristics & $\begin{array}{l}10 \text {-year ASCVD } \\
\text { risk Mean } \pm \text { SD }\end{array}$ & $\begin{array}{l}\text { Comparison 10-year } \\
\text { ASCVD between sub-group }\end{array}$ & $\begin{array}{l}\text { Spearman's rho }(\mathbf{r})^{* 2} \\
\text { correlation }\end{array}$ & Proportion n (\%) & OR (CI 95\%)*3 \\
\hline \multicolumn{6}{|l|}{ Total-C (mg/dl) } \\
\hline$<200$ (low) & $5.4 \pm 6.2$ & - & \multirow[t]{3}{*}{0.26} & $117(52.9)$ & 1.0 \\
\hline $200-239$ (moderate) & $8.6 \pm 7.9$ & $\mathrm{p}<0.01$ & & $71(32.1)$ & $3.1(1.6-6.0)$ \\
\hline$\geq 240$ (high) & $10.7 \pm 8.2$ & $p<0.01(p<0.01)$ & & 33 (14.9) & $6.3(1.3-14.3)$ \\
\hline \multicolumn{6}{|l|}{ HDL-C (mg/dl) } \\
\hline$<40$ (low) & $9.2 \pm 9.4$ & - & \multirow{3}{*}{-0.23} & $26(11.8)$ & 1.0 \\
\hline 40-60 (moderate) & $7.8 \pm 7.5$ & $\mathrm{p}=0.53$ & & $150(67.9)$ & $0.8(0.4-1.9)$ \\
\hline$>60$ (high) & $4.2 \pm 4.3$ & $\mathrm{p}=0.03(\mathrm{p}<0.01)$ & & $45(20.4)$ & $0.4(0.1-1.2)$ \\
\hline \multicolumn{6}{|l|}{ LDL-C $(\mathrm{mg} / \mathrm{dl})$} \\
\hline$<100$ (normal) & $5.6 \pm 5.8$ & - & \multirow[t]{5}{*}{0.19} & $40(18.1)$ & 1.0 \\
\hline 100-129 (near normal) & $6.3 \pm 7.3$ & $\mathrm{p}=0.57$ & & $85(38.5)$ & $0.7(0.3-1.6)$ \\
\hline 130-159 (borderline high) & $8.5 \pm 6.8$ & $p=0.02$ & & $54(24.4)$ & $1.7(0.7-4.1)$ \\
\hline 160-189 (high) & $9.4 \pm 9.4$ & $\mathrm{p}=0.03$ & & $24(10.9)$ & $1.8(0.6-5.3)$ \\
\hline >190 (very high) & $14.9 \pm 10.5$ & $\mathrm{p}=0.01(\mathrm{p}<0.01)$ & & $6(2.7)$ & $5.2(0.8-32.0)$ \\
\hline \multicolumn{6}{|l|}{ Triglyceride (mg/dl) } \\
\hline$<150$ (normal) & $6.0 \pm 6.5$ & - & \multirow[t]{4}{*}{0.35} & $148(67.0)$ & 1.0 \\
\hline 150-199 (borderline high) & $8.3 \pm 6.3$ & $\mathrm{p}=0.13$ & & $27(12.2)$ & $2.7(1.2-6.2)$ \\
\hline 200-499 (high) & $12.4 \pm 9.7$ & $p<0.01$ & & 33 (14.9) & $5.1(2.3-11.2)$ \\
\hline$\geq 500$ (very high) & n.a. & n.a. $(\mathrm{p}<0.01)$ & & $1(0.5)$ & n.a. \\
\hline \multicolumn{6}{|l|}{ Ratio triglyceride/HDL-C } \\
\hline$<2$ (ideal) & $4.6 \pm 5.0$ & - & \multirow{3}{*}{0.35} & $90(40.7)$ & 1.0 \\
\hline $2-<4$ (preferable) & $8.5 \pm 7.6$ & $\mathrm{p}<0.01$ & & $78(35.3)$ & $3.0(1.5-6.2)$ \\
\hline$\geq 4$ (high risk) & $11.3 \pm 9.2$ & $p<0.01(p<0.01)$ & & 41 (18.6) & $6.5(2.9-14.6)$ \\
\hline \multicolumn{6}{|l|}{ Ratio total-C/HDL-C } \\
\hline <3.5 (ideal) & $5.0 \pm 5.3$ & - & \multirow[t]{3}{*}{0.35} & 79 (35.7) & 1.0 \\
\hline $3.5-<5$ (preferable) & $7.3 \pm 7.5$ & $\mathrm{p}=0.01$ & & $102(46.2)$ & $1.2(0.6-2.3)$ \\
\hline$\geq 5$ (high risk) & $11.8 \pm 8.4$ & $\mathrm{p}<0.01(\mathrm{p}<0.01)$ & & $40(18.1)$ & $4.8(2.2-10.4)$ \\
\hline \multicolumn{6}{|l|}{ Ratio LDL-C/HDL-C } \\
\hline$<2.5$ (ideal) & $5.3 \pm 5.4$ & - & \multirow[t]{3}{*}{0.29} & $98(44.3)$ & 1.0 \\
\hline 2.5-3.5 (preferable) & $7.6 \pm 7.0$ & $\mathrm{p}=0.01$ & & 85 (38.5) & $1.6(0.8-3.0)$ \\
\hline$\geq 3.5$ (high) & $14.5 \pm 10.5$ & $\mathrm{p}<0.01(\mathrm{p}<0.01)$ & & $28(12.7)$ & $9.4(3.7-24.3)$ \\
\hline
\end{tabular}

CVD: Cardiovascular disease; 10-year ASCVD risk in percentage (\%). ${ }^{* 1}$ p value for comparison 10-year. ASCVD between sub-group using Mann-Whitney test and KruskalWallis test for the variables with 2 sub-groups and $>2$ sub-groups respectively; the lowest value in the sub-group as a control group. ${ }^{* 2}$ Spearman's rho correlation: 10 -year ASCVD risk with the variables. ${ }^{* 3} \mathrm{OR}(95 \% \mathrm{CI})$ : odds ratio (95\% confidence interval); the lowest cut point in the sub-group as a control group. ASCVD: Atherosclerotic cardiovascular disease, HDL-C: High-density lipoprotein-cholesterol, LDL-C: Low-density lipoprotein-cholesterol, Total-C: Total-cholesterol, SD: Standard deviation

premature ASCVD [17]. The finding from the previous study in the area showed the older age of the subjects had the more ASCVD risk factors [13]. The subjects in this study had the high level of BMI, and it is likely the signal of early ASCVD, higher risk of DM, hypertension, and hypercholesterolemia [18]. An investigation done in urban India showed that the earlier escalation of ASCVD risk factors at the younger age of 30-39 year [19]. The subjects in this study were from rural area, and the subject's environment was likely the secondary reason for the relatively low ASCVD risk.

The ASCVD risk indicated a higher proportion of subjects eligible for statin than another method [3]. It was found in the study that high proportion of subjects needed for statin. The finding was similar to the study done in Denmark with $42 \%$ subjects eligible for statin using the ASCVD risk score and only 6\% with the ESC/EAS guideline [20]. There was disagreement regarding the higher proportion of eligible for statin subjects based on ASCVD risk. A study criticized, the ASCVD caused the increased statin prescription frequency to subjects [10], whereas the other study supported the more statins based on ASCVD [2]. Furthermore, a study recommended the therapeutic lifestyle modification and/or pharmacology intervention reduce the atherosclerosis and suggested the preventive activities were costeffectiveness among subjects with or without DM [21]. Study in India showed the increase of physical activities with telephone counseling intervention in DM subjects [22]. Lifestyle modification should be considered as one method that could prevent ASCVD.

The subjects in this study had low awareness, and also did not initiate or continue the treatment with statin. This condition was likely related to the subjects' profile, i.e., low educational background discussed in the preceding study [13].
The prevalence of smoking, DM, SBP $\geq 140 \mathrm{mmHg}$ was at $52.9 \%, 10.4 \%$, and $39.8 \%$, respectively, and could be considered as high prevalence. Besides the BMI variable, all other non-lipid variables showed that higher risk of the subjects had the higher ASCVD risk. The subjects without hypertension therapy were considered lower risk and with significantly lower ASCVD risk. Most subjects had the inadequate therapy to control the high blood pressure or hypercholesterolemia the finding was not improved from the previous study $[12,13]$. The subjects were likely need pharmacology therapy. Atorvastatin and irbesartan in fixed-dose combination might be recommended for the patients with CVD with hyperlipidemias and hypertension. Both of medicines could increase endothelial function and reduce inflammatory [23].

Although the triglyceride was not belonged to one of the ASCVD variables, the 10-year risk of ASCDV had a significant association with triglyceride and ratio triglyceride/HDL-C. Many experts considered single parameter of lipid profile had better correlation with ASCVD, but a study showed that high ratio of triglycerides to HDL-C was likely to predict the existence of coronary heart disease [24].

The subjects in this study were Asian (non-White or non-African race $100 \%$ ) population, in the ASCVD risk method the Asian subjects were categorized as other than white and African. During the writing process of this study, there is no reference of the ASCVD risk information for Indonesian subjects. According to a reference, the ASCVD risk method appeared to overestimate absolute ASCVD risk as the observed events were less than predicted [8]. A similar finding in an external calibration on ASCVD risk, the result showed that the method in 15 cohort studies, reanalyzed studies, or external calibration overestimated the ASCVD risk in almost all studies. The studies included one cohort done among Malaysian whose population had similar genetics to Indonesian 
subjects [11]. After 3 years of PCE launching, a study suggested to recalibrate the PCE [25]. While one cohort in Europe preferred the ASCVD risk than SCORE from ESC/EAS guidelines due to the method had higher sensitivity and specificity for the eligibility for statin [20]. The ASCVD risk predicted from this study needs further investigation and comparison with the actual ASCVD risk observed among the Sleman District population.

\section{Limitation}

There were 15 subjects missed in the study because the ASCVD risk method could not cover the <40-year subjects. The sub-groups of gender, hypertension treatment, DM, and the lipid profiles had the different proportion in each sub-group because the study included all eligible subjects without matching the subjects in each group. The ASCVD risk from PCE was known to underestimate or overestimate the 10-year ASCVD risk for subjects from other than white and African American races, though the ASCVD risk model was likely give a better prediction in general population.

\section{CONCLUSIONS}

The 10-year ASCVD risk prediction of the subjects using the PCE method had the median score at $4.4(0.2-41.4)$, and the score was classified as low-risk of ASCVD. The ASCVD risk had the significant association with non-lipid profiles, total-C, and ratio total-C/HDL-C, triglyceride/HDL-C, and LDL-C/HDL-C.

\section{ACKNOWLEDGMENT}

We are thankful to Director General of Higher Education, Indonesian Ministry of Research, Technology and Higher Education of the Republic of Indonesia, for the financial support of the research grant with Penelitian Unggulan Perguruan Tinggi scheme year 2016-2017.

\section{REFERENCES}

1. Smith SC, Collins A, Ferrari R, Holmes DR, Logstrup S, McGhie DV, et al. WHF/AHA/ACCF/EHN/ESC presidential advisory our time: A call to save preventable death from cardiovascular disease (heart disease and stroke). J Am Coll Cardiol 2012;60:2343-8.

2. Piepoli MF, Hoes AW, Agewall S, Albus C, Brotons C, Catapano AL, et al. European guidelines on cardiovascular disease prevention in clinical practice; The $66^{\text {th }}$ joint task force of the European society of cardiology and other societies on cardiovascular disease prevention in clinical practice. Eur Heart J 2016;37:2315-81.

3. Guerra-Silva NM, Santucci FS, Moreira RC, Massao Tashima C, de Melo SC, Pereira LR, et al. Coronary disease risk assessment in men: Comparison between ASCVD Risk versus Framingham. Int J Cardiol 2017;228:481-7.

4. NCEP-ATP III. Expert panel on detection, evaluation, and treatment of high blood cholesterol in adults. Executive summary of the $3^{\text {rd }}$ report of the national Cholesterol education program (NCEP) expert panel on detection, evaluation, and treatment of high blood cholesterol in adults (Adult Treatment Panel III). J Am Med Assoc 2001;285(19):2486-97.

5. Stone NJ, Robinson JG, Lichtenstein AH, Bairey Merz CN, Blum CB, Eckel RH, et al. 2013 ACC/AHA guideline on the treatment of blood cholesterol to reduce atherosclerotic cardiovascular risk in adults: A report of the American college of cardiology/American Heart Association task force on practice guidelines. Circulation 2014:129 25 Suppl 2:S1-45.

6. Goff DC, Llyod-Jones DM, Bennett G, Coady S, D'Agostino RB, Gibbons R, et al. 2013 ACC/AHA guideline on the assessment of cardiovascular risk: A report of the American college of cardiology/ American Heart Association task force on practice guidelines.
Circulation 2013;00:000

7. Catapano AL, Graham I, De Backer G, Wiklund O, Chapman MJ, Drexel H, et al. $2016 \mathrm{ESC} / \mathrm{EAS}$ guidelines for the management of dyslipidemias. Eur Heart J 2016;37(39):2999-3058.

8. Chia YC, Lim HM, Ching SM. Validation of the pooled cohort risk score in an Asian population - A retrospective cohort study. BMC Cardiovasc Disord 2014;14:163.

9. American College of Cardiology (ACC). ASCVD Risk Estimator; 2016. Available from: http://www.tools.acc.org/ASCVD-Risk-estimator/. [Last accessed on 2017 Mar 10].

10. Preiss D, Kristensen SL. The new pooled cohort equations risk calculator. Can J Cardiol 2015;31(5):613-9.

11. Cook NR, Ridker PM. Calibration of the pooled cohort equations for atherosclerotic cardiovascular disease; An update. Ann Intern Med 2016;165:786-94.

12. Suhadi R, Linawati Y, Virginia DM, Setiawan CH. Early implementation of universal health coverage among hypertension subjects in Sleman district of Yogyakarta. Acta Med Indones 2015;47(4):311-9.

13. Suhadi R, Linawati Y, Wulandari ET, Virginia DM, Setiawan $\mathrm{CH}$. The metabolic disorder and cardiovascular risk among lower socioeconomic subjects in Yogyakarta - Indonesia. Asian J Pharm Clin Res 2017;10:367-2.

14. Gerstman B. Stat Primer (Version 7.0). Cha. 14. Correlation; 2016. Available from: http://www.sjsu.edu/faculty/gerstman/StatPrimer/ correlation.pdf. [Last accessed on 2017 Mar 20]

15. Jousilahti P, Vartiainen E, Tuomilehto J, Puska P. Sex, age, cardiovascular risk factors, and coronary heart disease: A prospective follow-up study of 14786 middle-aged men and women in Finland. Circulation 1999;99(9):1165-72.

16. Ameer KA, Mohammed SI. The Correlation between Framingham risk score and the clinical and biochemical parameters that measure functional disability and disease activity in Iraqi patients with rheumatoid arthritis. Int J Pharm Pharm Sci 2014;6:284-386.

17. Mancia G, Fargard R, Narkiewicz K, Redön J, Zanchetti A, Böhm $\mathrm{M}$, et al. ESH/ESC guidelines for the management of arterial hypertension. The task force for the management of arterial hypertension of the European society of hypertension (ESH) and of the European Society of Cardiology (ESC). J Hypertens 2013;31(7):1281-357

18. Bays HE, Chapman RH, Grandy S. SHIELD investigators' group. The relationship of body mass index to diabetes mellitus, hypertension and dyslipidemia: Comparison of data from two national surveys. Int J Clin Pract 2007;61(5):737-47

19. Gupta R, Misra A, Vikram NK, Kondal D, Gupta SS, Agrawal A, et al. Younger age of escalation of cardiovascular risk factors in Asian Indian subjects. BMC Cardiovasc Disord 2009;9:28.

20. Mortensen MB, Nordestgaard BG, Afzal S, Falk E. ACC/AHA guidelines superior to ESC/EAS guidelines for primary prevention with statins in non-diabetic Europeans: The copenhagen general population study. Eur Heart J 2017;38:586-94.

21. Ayuttaya KS, Ayuttaya SS. Cost-effectiveness analysis of statin monotherapy regimen in outpatient management of dyslipidemia in patients with diabetes mellitus. Songklanakarin J Sci Technol 2014;36(6):675-81.

22. Dhaliwali C, Erinmacpherson, Richardson J. Effectiveness of telephone-delivered interventions for increasing physical activity levels in persons with Type 2 diabetes or hypertension: A systematic review. J Crit Rev 2015;2(4):6-11

23. Virani P, Sojitra R, Raj H, Vineet J. A review on Irbesartan co administered with atorvastatin for the treatment of cardiac risk. J Crit Rev 2014;1:25-8.

24. da Luz PL, Favarato D, Faria-Neto Jr JR, Lemos P, Chagas AC. High ratio of triglycerides to HDL-cholesterol ratio predicts extensive coronary disease. Clin Sci 2008;63(4):427-32.

25. Ridker PM, Cook NR. The pooled cohort equations 3 years on buiding a stronger foundation. Circulation 2016;134(23):1789-1 